\title{
In Situ Spectroscopic Studies of Highly Transparent Nanoparticle Dispersions Enable Assessment of Trithiocarbonate Chain-End Fidelity during RAFT Dispersion Polymerization in Nonpolar Media
}

\author{
Erik J. Cornel, ${ }^{\dagger}$ Sandra van Meurs, ${ }^{\dagger}$ Timothy Smith, ${ }^{\ddagger}$ Paul S. O’Hora, ${ }^{\ddagger}$ and Steven P. Armes* ${ }^{* \dagger \odot}$ \\ ${ }^{\dagger}$ Dainton Building, Department of Chemistry, University of Sheffield, Brook Hill, Sheffield, South Yorkshire S3 7HF, United \\ Kingdom \\ ${ }^{\ddagger}$ Lubrizol Ltd., Nether Lane, Hazelwood, Derbyshire DE56 4AN, United Kingdom
}

Supporting Information

\begin{abstract}
We report the synthesis of highly transparent poly(stearyl methacrylate)-poly(2,2,2-trifluoroethyl methacrylate) (PSMAPTFEMA) diblock copolymer nanoparticles via polymerization-induced self-assembly (PISA) in nonpolar media at $70{ }^{\circ} \mathrm{C}$. This was achieved by chain-extending a PSMA precursor block via reversible additionfragmentation chain transfer (RAFT) dispersion polymerization of TFEMA in $n$-tetradecane. This $n$-alkane has the same refractive index as the PTFEMA core-forming block at $70{ }^{\circ} \mathrm{C}$, which ensures high light transmittance when targeting $33 \mathrm{~nm}$ spherical nanoparticles. Such isorefractivity enables visible absorption spectra to be recorded with

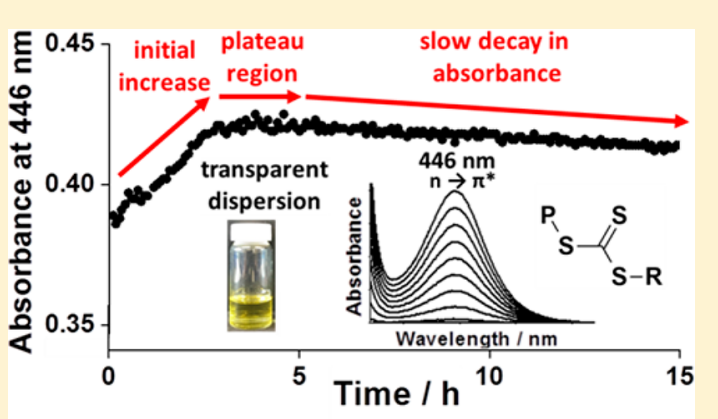
minimal light scattering even at $30 \% \mathrm{w} / \mathrm{w}$ solids. However, in situ monitoring of the trithiocarbonate RAFT end-groups during PISA requires selection of a weak $\mathrm{n} \rightarrow \pi^{*}$ band at $446 \mathrm{~nm}$. Conversion of TFEMA into PTFEMA causes a contraction in the reaction solution volume, leading to an initial increase in absorbance that enables the kinetics of polymerization to be monitored via dilatometry. At 98\% TFEMA conversion, this 446 $\mathrm{nm}$ band remains constant for $2 \mathrm{~h}$ at $70{ }^{\circ} \mathrm{C}$, indicating surprisingly high RAFT chain-end fidelity (and hence pseudoliving character) under monomer-starved conditions. In situ ${ }^{19} \mathrm{~F}$ NMR spectroscopy studies provide evidence for (i) the onset of micellar nucleation, (ii) solvation of the nanoparticle cores by TFEMA monomer, and (iii) surface plasticization of the nanoparticle cores by $n$-tetradecane at $70{ }^{\circ} \mathrm{C}$. Finally, the kinetics of RAFT chain-end removal can be conveniently monitored by in situ visible absorption spectroscopy: addition of excess initiator at $70{ }^{\circ} \mathrm{C}$ causes complete discoloration of the dispersion, with small-angle X-ray scattering studies confirming no change in nanoparticle morphology under these conditions.
\end{abstract}

\section{INTRODUCTION}

Block copolymer self-assembly in solution has become a wellestablished route for accessing a wide range of organic nanoparticles of varying size, morphology, and surface chemistry. ${ }^{1,2}$ One of the most powerful and versatile means of preparing functional block copolymers from various vinyl monomers is reversible addition-fragmentation chain transfer (RAFT) polymerization. ${ }^{3-6}$ This controlled radical polymerization technique provides good control over the molecular weight distribution and offers sufficient pseudoliving character to enable the synthesis of well-defined diblock copolymers. ${ }^{7-20}$ Self-assembly is traditionally achieved via post-polymerization processing, but over the past decade many research groups have demonstrated that polymerization-induced self-assembly (PISA) offers decisive advantages for the efficient synthesis of diblock copolymer nanoparticles directly in a wide range of solvents (e.g., water, polar solvents, nonpolar solvents, ionic liquids, etc.). ${ }^{21-25}$ When PISA is conducted via RAFT dispersion polymerization a soluble homopolymer precursor is chain-extended using a second miscible monomer, which forms an insoluble block when polymerized. Self-assembly occurs when this growing insoluble block reaches a certain critical degree of polymerization, with sterically stabilized diblock copolymer spheres, worms, or vesicles being formed depending on the relative block volume fractions and copolymer concentration. ${ }^{10,23,24,26-37}$

RAFT chain transfer agents (CTAs) are organosulfur compounds that can be utilized for chain-end functionalization. ${ }^{38-41}$ For optimal control, RAFT chain-ends should remain stable during RAFT polymerization, but it is wellknown that RAFT end-groups are gradually lost either under monomer-starved conditions ${ }^{7,42-44}$ or in the presence of water. ${ }^{45,46}$ Moreover, such CTAs are colored, malodorous, and potentially toxic, so their quantitative removal is often desired for potential applications. ${ }^{47,48}$ Fortunately, RAFT chain-ends can be readily cleaved using reagents such as amines, ${ }^{49}$ ozone, ${ }^{47} \mathrm{H}_{2} \mathrm{O}_{2}$, ${ }^{50}$ or excess radical initiator. ${ }^{51}$

Semsarilar et al. examined the stability of trithiocarbonate RAFT end-groups for the RAFT dispersion polymerization of

Received: July 26, 2018

Published: September 25, 2018 
2,2,2-trifluoroethyl methacrylate (TFEMA) in ethanol using either a poly(methacrylic acid) (PMAA) or a poly(2(dimethylamino)ethyl methacrylate) (PDMA) chain transfer agent (CTA). ${ }^{42}$ The growing PTFEMA block became insoluble in ethanol and hence formed the nanoparticle cores, with the PMAA or PDMA chains acting as the steric stabilizer. Semifluorinated polymers typically exhibit relatively low refractive indices. ${ }^{52}$ In this particular example, the refractive index of PTFEMA (1.41) is reasonably close to that of ethanol (1.36), which results in a weakly scattering dispersion. Periodic sampling of the reaction solution for such a dispersion polymerization followed by dilution enabled UV spectra to be recorded, and hence the RAFT chain-end fidelity to be assessed. A slow decay in RAFT chain-end fidelity was observed over $24 \mathrm{~h}$ at $70{ }^{\circ} \mathrm{C}$ by monitoring the absorption maximum corresponding to the trithiocarbonate end-groups at $305 \mathrm{~nm}$. Approximately $43 \%$ end-groups were lost during RAFT solution polymerization of TFEMA in THF at $70{ }^{\circ} \mathrm{C}$, whereas only $27 \%$ end-groups were degraded during the corresponding RAFT dispersion polymerization of TFEMA in ethanol under the same conditions. Thus, the latter heterogeneous polymerization offers greater pseudoliving character (and a faster rate of polymerization) than the equivalent homogeneous polymerization. ${ }^{53}$ However, in situ spectroscopic studies were not feasible for this PISA formulation, partly because the dispersions were not sufficiently isorefractive and also because the UV signal arising from the RAFT CTA chain-ends was far too intense (molar extinction coefficient, $\varepsilon_{\max } \approx 1.5 \times 10^{4} \mathrm{dm}^{3} \mathrm{~mol}^{-1} \mathrm{~cm}^{-1}$ ).

Herein, we report the RAFT dispersion polymerization of TFEMA in $n$-tetradecane to afford poly(stearyl methacrylate)poly(2,2,2-trifluoroethyl methacrylate) (PSMA-PTFEMA) spherical nanoparticles of $33 \mathrm{~nm}$ diameter. These nanoparticles are almost perfectly isorefractive with the solvent at the reaction temperature of $70{ }^{\circ} \mathrm{C}$, which enables high-quality visible absorption spectra to be recorded in situ without any interference from particle scattering. This allows the RAFT chain-end fidelity to be conveniently monitored throughout the polymerization, even when preparing such nanoparticles at $30 \% \mathrm{w} / \mathrm{w}$ solids. Subsequently, removal of the RAFT endgroups can be monitored in situ after addition of excess initiator at $70{ }^{\circ} \mathrm{C}$. Moreover, in situ ${ }^{19} \mathrm{~F}$ NMR spectroscopy studies provide useful insights with regard to both the PISA mechanism and the ingress of hot solvent within the nanoparticle cores at $70{ }^{\circ} \mathrm{C}$. Finally, such highly transparent dispersions may offer new opportunities for further scientific studies in the field of colloid science. ${ }^{54-56}$

\section{RESULTS AND DISCUSSION}

In initial scouting experiments, poly(stearyl methacrylate)poly(2,2,2-trifluoroethyl methacrylate) (PSMA-PTFEMA) diblock copolymer nanoparticles were prepared via RAFT dispersion polymerization in either $n$-tetradecane or $n$ dodecane (see Figure 1 and Figure S1). As expected, the PTFEMA block became insoluble at a certain critical degree of polymerization (DP) as it grows from the soluble PSMA block; this leads to micellar nucleation and eventually the formation of sterically stabilized nanoparticles. Such PISA syntheses enable high monomer conversions to be achieved (>98\%) and can be performed at relatively high copolymer concentration (30\% w/w in this particular case). ${ }^{29}$

In the present study, we sought to identify suitable reaction conditions to produce highly transparent dispersions. This is

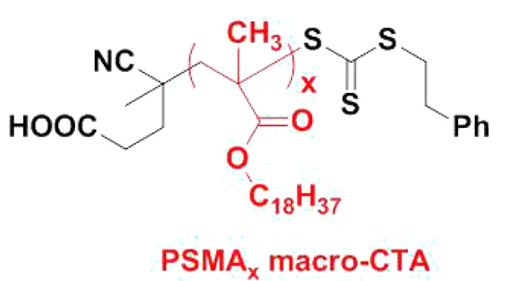<smiles>C=C(C)C(=O)OCC(C)(F)F</smiles>

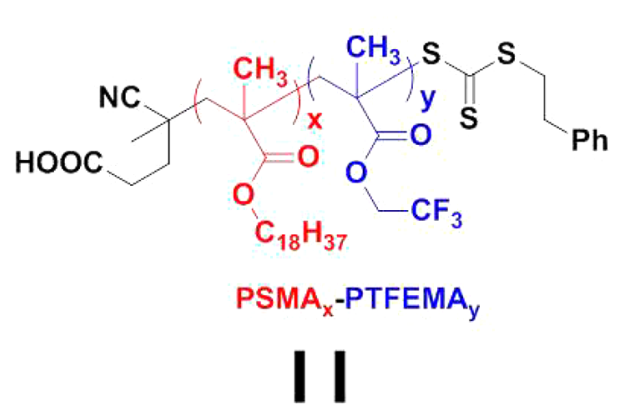

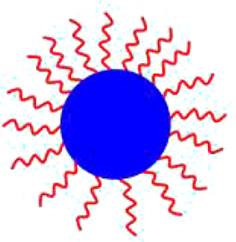

Figure 1. A PSMA $x$ homopolymer precursor is chain-extended via RAFT dispersion polymerization of TFEMA in either $n$-dodecane or $n$-tetradecane at 70 or $90{ }^{\circ} \mathrm{C}$. The growing PTFEMA block becomes insoluble at a certain critical DP, leading to in situ self-assembly to form spherical PSMA - PTFEMA $_{y}$ nanoparticles.

because we wished to utilize in situ visible absorption spectroscopy to monitor the fidelity of the trithiocarbonate RAFT end-groups during RAFT dispersion polymerization. It is essential that particle scattering is eliminated for such studies. Otherwise, Tyndall scattering results in a rising baseline at shorter wavelengths which prevents reliable quantification of the spectra. ${ }^{57}$ PTFEMA was selected as the core-forming block because this semifluorinated polymer has a relatively low refractive index $\left(1.418\right.$ at $\left.20{ }^{\circ} \mathrm{C}\right) .{ }^{52}$ PTFEMA is insoluble in all linear $n$-alkanes, and this homologous series of solvents offers an appropriate range of refractive indices. ${ }^{58}$ Thus, it should be feasible to obtain a reasonably good match to the PTFEMA refractive index by simply selecting an appropriate $n$-alkane as the solvent for the PISA synthesis. ${ }^{59}$ However, this approach is complicated by the temperature dependence of the refractive index for both the PTFEMA and the $n$-alkane. Figure 2 shows the transmittance versus temperature plots recorded for PSMA $_{32}-$ PTFEMA $_{490}$ nanoparticles prepared via PISA in $n$-dodecane and $n$-tetradecane, respectively. The former solvent provides a highly transparent dispersion for PSMA $_{32}-$ PTFEMA $_{490}$ nanoparticles of $235 \pm 77$ $\mathrm{nm}$ diameter at $30{ }^{\circ} \mathrm{C}$ (see Figure $\mathrm{S} 1$ ), but a relatively turbid dispersion at higher temperatures (e.g., see the inset digital image recorded at $90{ }^{\circ} \mathrm{C}$ ). Because RAFT polymerizations are typically performed at $60-90{ }^{\circ} \mathrm{C}$, such turbidity prevents in 


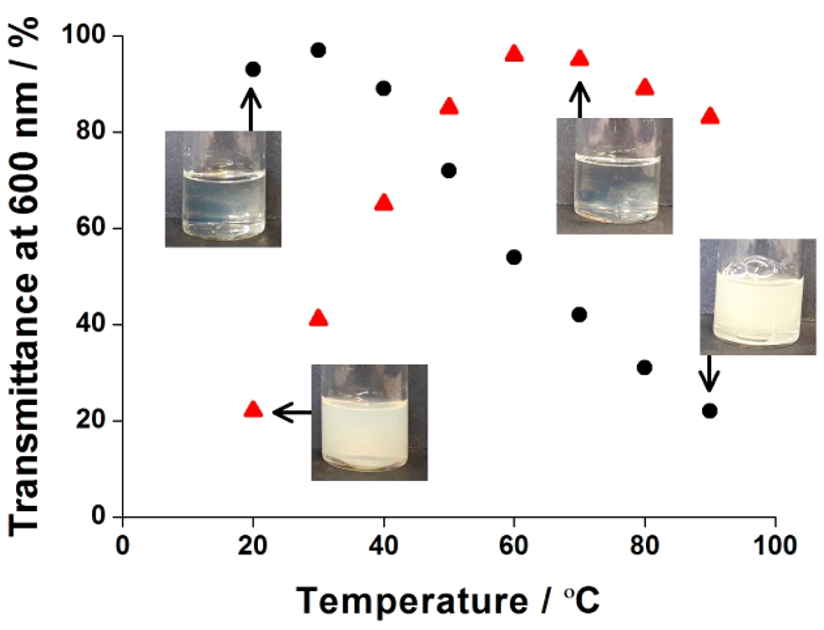

Figure 2. Transmittance (at $600 \mathrm{~nm}$ ) versus temperature plots recorded for $\mathrm{PSMA}_{32}-\mathrm{PTFEMA}_{490}$ spherical nanoparticles prepared via RAFT dispersion polymerization of TFEMA in either $n$-dodecane $(\boldsymbol{C})$ or $n$-tetradecane $(\boldsymbol{\Lambda})$ at $30 \% \mathrm{w} / \mathrm{w}$ solids. For a given particle size and concentration, the refractive index difference between the PTFEMA nanoparticle cores and the solvent (either $n$-dodecane or $n$-tetradecane) determines the turbidity of the dispersion.

situ visible absorption spectroscopy studies from being performed during PISA syntheses conducted in this solvent. In contrast, similar-sized PSMA $_{32}-$ PTFEMA $_{490}$ nanoparticles $(240 \pm 36 \mathrm{~nm}$ diameter $)$ prepared in $n$-tetradecane are turbid at ambient temperature but become highly transparent at elevated temperature, with a minimum in turbidity being observed at around $60-70{ }^{\circ} \mathrm{C}$. It is perhaps worth emphasizing the remarkably subtle nature of this refractive index matching problem: the refractive indices of $n$-dodecane and $n$ tetradecane at $20{ }^{\circ} \mathrm{C}$ are 1.421 and 1.429 , respectively. In view of these initial observations, we elected to perform PISA syntheses of PSMA-PTFEMA nanoparticles in $n$-tetradecane at $70{ }^{\circ} \mathrm{C}$. For spherical nanoparticles, it is well-known that the scattered light intensity scales as the sixth power of the particle radius. ${ }^{60}$ Thus, shorter DPs were targeted for the PTFEMA block to reduce the nanoparticle core diameter and hence further minimize the dispersion turbidity. More specifically, PSMA $_{12}-$ PTFEMA $_{100}$ nanoparticles were targeted at a copolymer concentration of $30 \% \mathrm{w} / \mathrm{w}$, and this PISA formulation forms the basis of the rest of this article.

The very high transmittance ( $>99 \%$ ) observed for $\mathrm{PSMA}_{12}-$ PTFEMA $_{98}$ nanoparticles in $n$-tetradecane at $70{ }^{\circ} \mathrm{C}$ is sufficient to enable high-quality visible absorption spectra to be recorded with minimal interference from particle scattering. In principle, this can be achieved by using a commercial all-quartz UVvisible probe (Figure S2) and a dedicated UV-visible spectrometer. In practice, such in situ measurements also require a sufficiently weak absorption band to avoid signal saturation when monitoring PISA syntheses performed in concentrated solution $(30 \% \mathrm{w} / \mathrm{w})$. Fortunately, the wellknown "forbidden" absorption band at $446 \mathrm{~nm}$ associated with trithiocarbonate RAFT end-groups proved to be suitable for the purpose of this study. ${ }^{61}$ Shi and co-workers assigned this spectral feature to an $\mathrm{n} \rightarrow \pi^{*}$ transition and used it to monitor the stability of trithiocarbonate chain-ends during the visible light-mediated RAFT aqueous solution polymerization of 2-(Nacryloyloxyethyl) pyrrolidone (NAEP) at $25{ }^{\circ} \mathrm{C}$ using a watersoluble photoinitiator. ${ }^{61}$ However, as far as we are aware, this band has not been exploited to monitor the progress of any

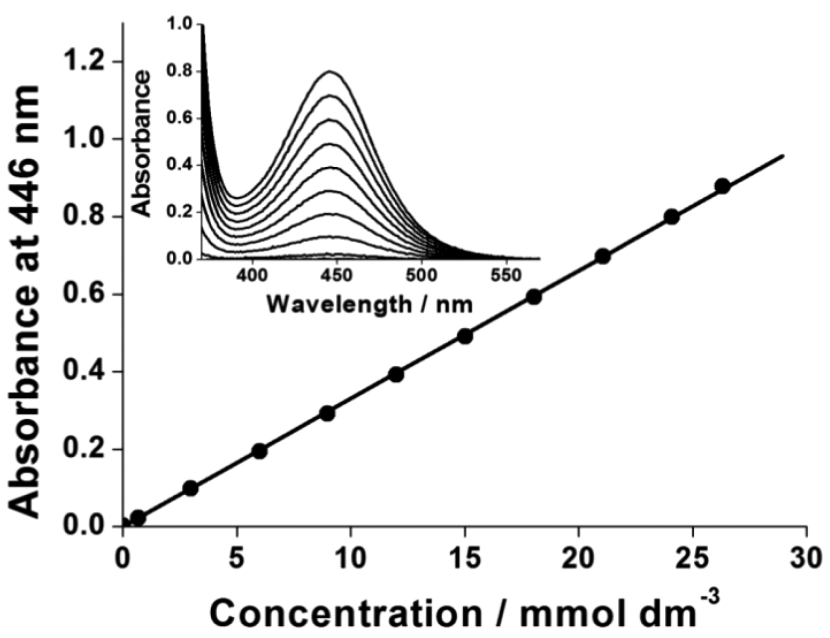

Figure 3. Beer-Lambert calibration curve constructed for the trithiocarbonate-based $\mathrm{PSMA}_{12}$ precursor block (inset shows the associated visible absorption spectra) using its relatively weak $\mathrm{n} \rightarrow \pi^{*}$ band at $\lambda_{\max }=446 \mathrm{~nm}$, for which $\varepsilon$ is calculated to be $33.21 \pm 0.14$ $\mathrm{dm}^{3} \mathrm{~mol}^{-1} \mathrm{~cm}^{-1}$. This PSMA 12 RAFT agent also exhibits a much more intense band at $305 \mathrm{~nm} .{ }^{42}$ However, this latter spectral feature is too strong to allow in situ spectroscopic studies during RAFT dispersion polymerizations performed at $30 \% \mathrm{w} / \mathrm{w}$.

heterogeneous formulations such as RAFT dispersion polymerization, for which isorefractivity is essential. A Beer-Lambert calibration plot for the trithiocarbonate-based PSMA precursor block used in this study indicated that this weak $446 \mathrm{~nm}$ band had a molar extinction coefficient of $33.21 \pm$ $0.14 \mathrm{dm}^{3} \mathrm{~mol}^{-1} \mathrm{~cm}^{-1}$ (see Figure 3). For comparison, the wellknown UV band at $305-310 \mathrm{~nm}$ associated with trithiocarbonates has a much higher molar extinction coefficient of $\sim 1.5$ $\times 10^{4} \mathrm{dm}^{3} \mathrm{~mol}^{-1} \mathrm{~cm}^{-1}$. 42,62

Spherical PSMA $12-$ PTFEMA $_{98}$ nanoparticles were prepared in $n$-tetradecane at $30 \% \mathrm{w} / \mathrm{w}$, and this isorefractive PISA formulation was studied using in situ visible absorption spectroscopy. Preliminary experiments yielded very noisy data, because gas bubbles associated with the thermal decomposition of the lauroyl peroxide initiator led to sporadic light scattering (and hence anomalous absorbance values). Fortunately, this technical problem was solved by inclining the $\mathrm{UV}$-visible quartz probe at an angle of approximately $45^{\circ}$ so that the rising gas bubbles were much less likely to interfere with the light beam (see Figure S2). Originally, we had expected to see a gradual decay in the $446 \mathrm{~nm}$ absorption band during polymerization due to the degradation of RAFT endgroups. $7,42-44$ In fact, a significant increase in absorbance at $446 \mathrm{~nm}$ was observed over the first $3 \mathrm{~h}$ of the TFEMA polymerization (Figure 4), followed by a plateau region where the absorbance of this band remained approximately constant for at least $2 \mathrm{~h}$ at $70{ }^{\circ} \mathrm{C}$. Initially, we were puzzled by the unexpected increase in absorbance within the first $3 \mathrm{~h}$. Eventually, we realized that this was directly related to the significant volume contraction that occurs during the conversion of TFEMA monomer $\left(\rho=1.18 \mathrm{~g} \mathrm{~cm}^{-3}\right.$ at 20 $\left.{ }^{\circ} \mathrm{C}\right)^{63}$ into PTFEMA $\left(\rho=1.47 \mathrm{~g} \mathrm{~cm}^{-3}\right.$ at $\left.20{ }^{\circ} \mathrm{C}\right),{ }^{64}$ which leads to an increase in the RAFT end-group concentration. Solution density measurements were performed before and after the TFEMA polymerization to confirm this interpretation. The theoretical change in absorbance due to volumetric contraction, $\Delta A$, is calculated to be 0.032 from the densities of the initial and final dispersions at $70{ }^{\circ} \mathrm{C}$; see eq $\mathrm{S} 1$. The 

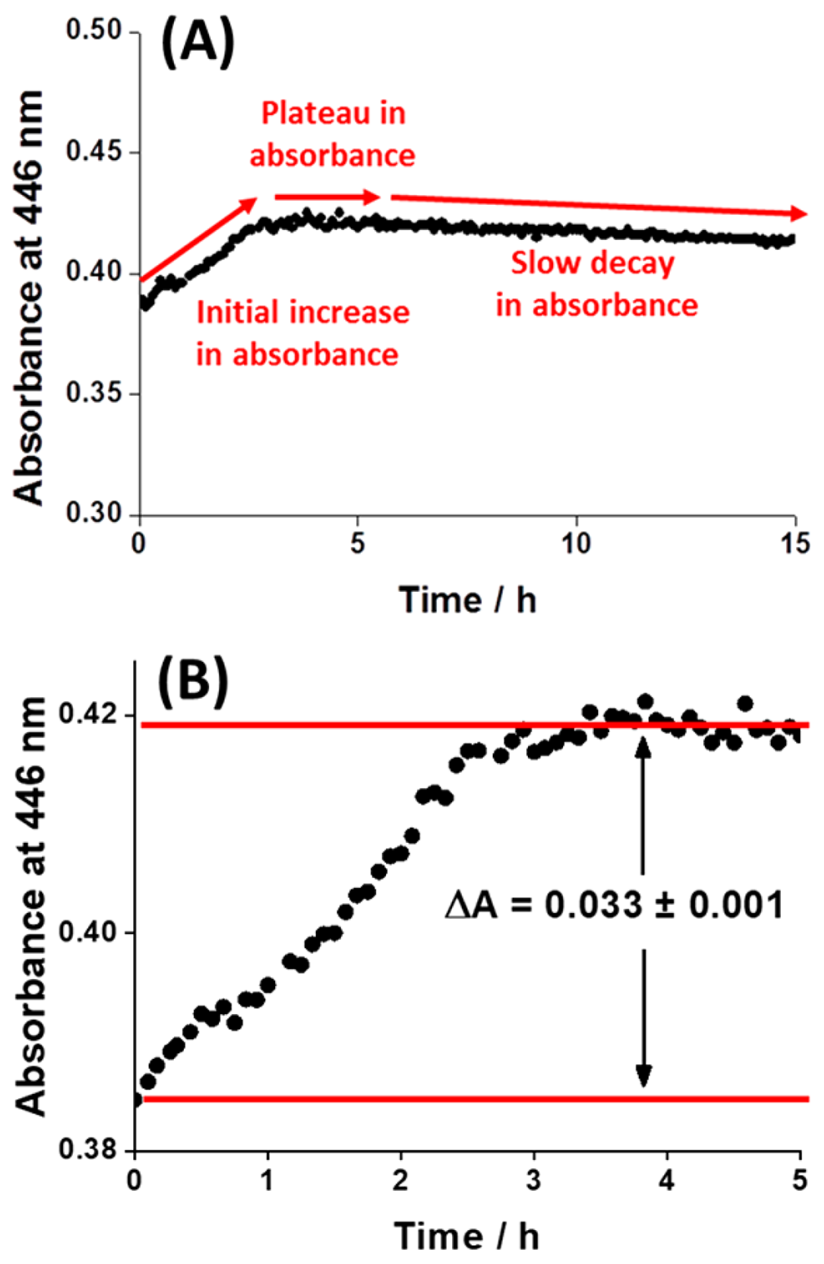

Figure 4. (a) Typical absorbance versus time plot obtained via in situ visible absorption spectroscopy studies of the RAFT dispersion polymerization of TFEMA using a $\mathrm{PSMA}_{12}$ macro-CTA at $70{ }^{\circ} \mathrm{C}$, where the weak absorption band at $446 \mathrm{~nm}$ is assigned to the trithiocarbonate RAFT end-groups. The constant absorbance observed after approximately $3 \mathrm{~h}$ indicates excellent RAFT chainend stability under monomer-starved conditions, with only a slow decay in absorbance observed between 5 and $15 \mathrm{~h}$. (b) A significant volume contraction occurs on converting TFEMA $\left(\rho=1.18 \mathrm{~g} \mathrm{~cm}^{-3}\right)$ into PTFEMA $\left(\rho=1.47 \mathrm{~g} \mathrm{~cm}^{-3}\right)$, which leads to the observed increase in absorbance. These data can be used to conveniently monitor the polymerization kinetics via dilatometry (see Figure 5).

experimentally observed increase in absorbance (0.033 \pm 0.001) indicated in Figure 4 is equal to this theoretical value within experimental error. Such a dilatometric effect is wellknown in polymer science and has been previously utilized to monitor the kinetics of polymerization. ${ }^{65,66}$ Hence, we examined whether the RAFT end-group absorption band at $446 \mathrm{~nm}$ could be used to monitor the kinetics of such an isorefractive RAFT dispersion polymerization.

The raw absorbance data shown in Figure 4 can be converted into fractional TFEMA conversions using eq S2. The resulting conversion versus time curve determined by in situ visible absorption spectroscopy (Figure 5a) is in good agreement with kinetic data obtained by ${ }^{19} \mathrm{~F}$ NMR studies conducted in $\mathrm{CDCl}_{3}$, which is a good solvent for both the PSMA and the PTFEMA blocks and hence ensures molecular dissolution of the nanoparticles prior to their analysis (Figure $5 B)$. Aliquots were periodically taken during the TFEMA polymerization, with $96 \%$ TFEMA conversion being achieved
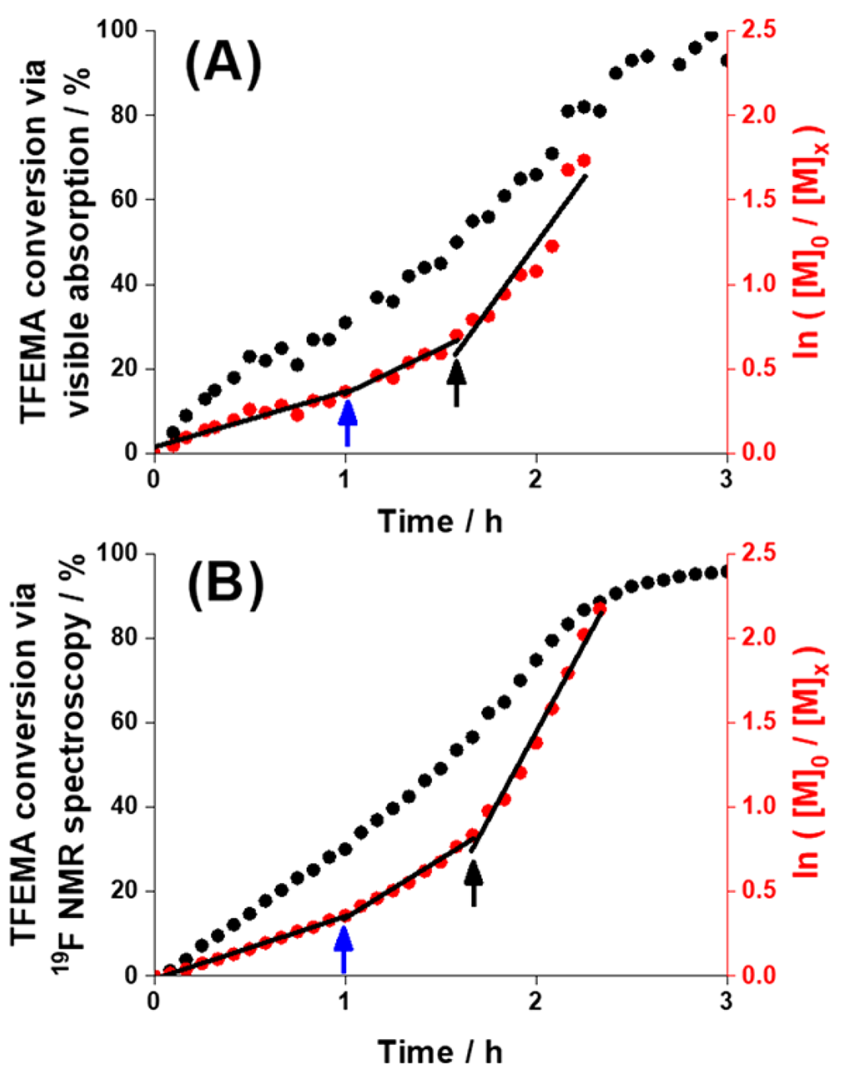

Figure 5. Conversion versus time curves and associated semilogarithmic plots for the RAFT dispersion polymerization of TFEMA at $70{ }^{\circ} \mathrm{C}$ using a $\mathrm{PSMA}_{12}$ precursor to produce a $30 \% \mathrm{w} / \mathrm{w}$ dispersion of sterically stabilized spherical nanoparticles. Remarkably similar reaction kinetics were observed by (a) in situ visible absorption spectroscopy using the weak $446 \mathrm{~nm}$ band assigned to the trithiocarbonate end-groups and (b) periodic sampling of the polymerization, followed by ${ }^{19} \mathrm{~F}$ NMR spectroscopy analysis of the quenched aliquots. The TFEMA conversion was $98 \%$ after $3 \mathrm{~h}$, indicating a final $\mathrm{PSMA}_{12}-\mathrm{PTFEMA}_{98}$ composition. Changes in reaction rate are indicated by blue and black arrows.

within $3 \mathrm{~h}$. The corresponding semilogarithmic kinetic plots revealed three distinct linear regimes in each case. A discernible change in the rate of polymerization is observed after $1 \mathrm{~h}$, which corresponds to the onset of micellar nucleation (Figure 5, see blue arrows). This occurs at approximately $20 \%$ TFEMA conversion, which suggests that the growing PTFEMA chains become insoluble in the polymerizing mixture when they reach a critical DP of approximately 20, with further polymerization occurring within monomer-swollen micelles. The higher local monomer concentration leads to an approximate two-fold increase in the rate of polymerization, as judged by the change in gradient (Figure 5). Similar observations have been reported for other RAFT dispersion polymerizations. ${ }^{29,67}$ Normally, such micellar nucleation is accompanied by solution turbidity, but this cannot be observed for the isorefractive dispersions studied herein. Instead, smallangle X-ray scattering (SAXS) studies were performed at various time points to confirm the onset of micellization after approximately $1 \mathrm{~h}$ (Figure S3). Unexpectedly, both in situ visible absorption spectroscopy and ${ }^{19} \mathrm{~F}$ NMR studies also indicate a second change in slope, with a further three-fold rate enhancement being observed after $115 \mathrm{~min}$ (Figure 5, see black arrows). This is tentatively interpreted as a switch from relatively loose nascent micelles to the formation of more 
compact micelles, for which the local monomer concentration is somewhat higher because the micelle cores contain less solvent.

Returning to Figure 4, the above explanation for the initial increase in absorbance has an important consequence: the constant absorbance observed in the 3-5 h interval implies minimal loss of RAFT chain-ends under monomer-starved conditions at $70{ }^{\circ} \mathrm{C}$, at least for this $2 \mathrm{~h}$ time period. In contrast, $27 \%$ loss of RAFT chain-ends was reported by Semsarilar and co-workers for the RAFT dispersion polymerization of TFEMA in ethanol using the same RAFT agent at the same temperature. ${ }^{42}$ However, the rate of polymerization of TFEMA was significantly slower for this latter PISA formulation, with $24 \mathrm{~h}$ being required for $91 \%$ conversion. Finally, it is perhaps worth emphasizing that gradual loss of the RAFT chain-ends is observed when the present PISA formulation is monitored over significantly longer time scales (5-15 h), as originally expected (see Figure 4).

To further investigate the polymerization kinetics, in situ ${ }^{19} \mathrm{~F}$ NMR spectroscopy studies were performed. An NMR tube was loaded with the degassed reaction solution along with an inner NMR tube containing a solution of trifluorotoluene in $d_{8}$-toluene, which served as an external standard. Typical ${ }^{19} \mathrm{~F}$ NMR spectra recorded during the polymerization of TFEMA when targeting PSMA $_{12}-$ PTFEMA $_{100}$ nanoparticles at $70{ }^{\circ} \mathrm{C}$ are shown in Figure 6a (also see Figures S4 and S5). The in situ ${ }^{19}$ F NMR spectra shown in Figure 6 exhibit three distinct features: a TFEMA monomer triplet at $-75.3 \mathrm{ppm}$ (with satellite triplets at -75.1 and $-75.7 \mathrm{ppm}$ ), a broad PTFEMA signal at $-74.6 \mathrm{ppm}$, and the trifluorotoluene signal used as an external standard at $-63.7 \mathrm{ppm}$ (Figure S4). As expected, there is a gradual reduction in the TFEMA signal during the course of the polymerization, while the PTFEMA signal intensity progressively increases. After normalization with respect to the external standard, the PTFEMA signal intensity is shown as a function of TFEMA conversion in Figure 6b. This latter signal increases initially before reaching a maximum after approximately $1 \mathrm{~h}$, which corresponds to the onset of micellar nucleation. After nucleation, the growing nascent micelles become enriched with unreacted TFEMA monomer, which accounts for the two-fold rate enhancement observed in Figure 5. The reduced mobility of the growing PTFEMA chains within the micelle cores accounts for the gradual broadening and apparent attenuation of the ${ }^{19} \mathrm{~F}$ NMR signal observed at $-74.6 \mathrm{ppm}$ in Figure 6. However, the integrated peak area for this polymer signal continues to increase after nucleation, which enables determination of the TFEMA conversion. A third distinct kinetic regime is observed after approximately 43\% TFEMA conversion (Figure 5), which indicates a PTFEMA DP of 43. Interestingly, this corresponds to a change in the rate of attenuation of the PTFEMA signal intensity, which thereafter proceeds relatively slowly up to $98 \%$ conversion (Figure 6b). This suggests that there is little or no further expulsion of $n$-tetradecane solvent from the growing nanoparticle cores from this point until the TFEMA polymerization ceases.

To examine the extent of core solvation for the final PSMA $_{12}-$ PTFEMA $_{98}$ nanoparticles, a series of ${ }^{19} \mathrm{~F}$ NMR spectra were recorded from 10 to $90{ }^{\circ} \mathrm{C}$ (Figure 7). The increase in intensity for the broad polymer signal at $-74.6 \mathrm{ppm}$ indicates plasticization of the PTFEMA nanoparticle cores by the hot $n$-tetradecane (note that the sharp signal at $-75.3 \mathrm{ppm}$ is assigned to approximately $2 \%$ residual TFEMA monomer).
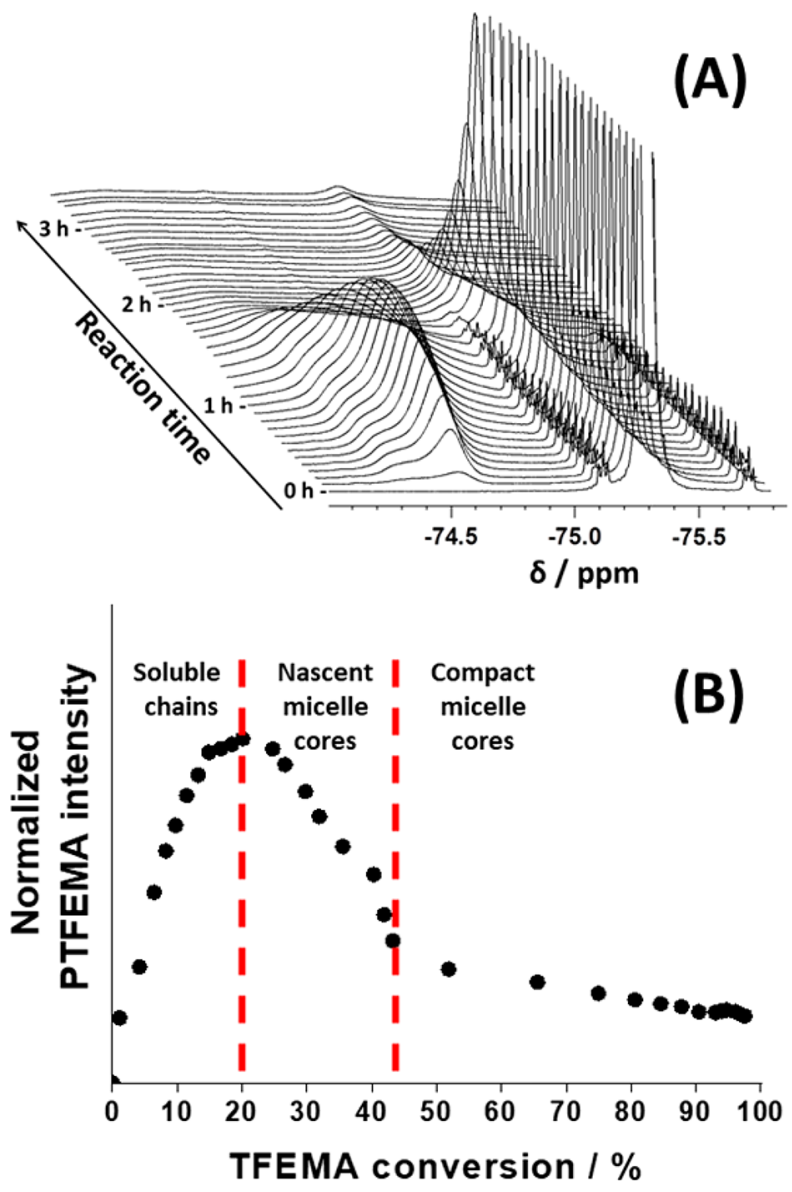

Figure 6. (a) ${ }^{19} \mathrm{~F}$ NMR spectra recorded at $70{ }^{\circ} \mathrm{C}$ during the in situ polymerization of TFEMA when targeting PSMA $_{12}-$ PTFEMA $_{100}$ nanoparticles at $30 \% \mathrm{w} / \mathrm{w}$. The intensity of the monomer triplet signal at $-75.3 \mathrm{ppm}$ decreases during polymerization. (b) The corresponding polymer signal at $-74.6 \mathrm{ppm}$ initially increases before reaching a maximum after $1 \mathrm{~h}$, which indicates the onset of micellar nucleation. This occurs at 20\% TFEMA conversion, which suggests that the growing PTFEMA chains become insoluble at a critical DP of $\sim 20$ under these conditions.

These results suggest that RAFT end-group removal should be feasible at elevated temperature, because such solvation should enhance the diffusion of reagent into the nanoparticle cores.

In principle, chemically stable RAFT chain-ends should offer enhanced control over the molecular weight distribution. However, RAFT chain-end removal is desirable for various potential applications. ${ }^{40,68}$ In this context, Jesson and coworkers recently reported that dithiobenzoate chain-ends can be readily removed from a range of diblock copolymer nanoparticles in aqueous media by adding a five-fold excess of $\mathrm{H}_{2} \mathrm{O}_{2}$ at $70{ }^{\circ} \mathrm{C} .{ }^{50}$ However, we are not aware of any reports of the removal of RAFT chain-ends from diblock copolymer nanoparticles prepared in nonpolar media. Thus, we examined the effect of adding an excess of lauroyl peroxide initiator to a $30 \% \mathrm{w} / \mathrm{w}$ dispersion of PSMA $_{12}-$ PTFEMA $_{98}$ nanoparticles in $n$-tetradecane at $70{ }^{\circ} \mathrm{C}$. The highly transparent nature of this dispersion enabled the kinetics of RAFT chain-end removal to be conveniently monitored in situ using visible absorption spectroscopy (Figure 8). The initial pale yellow dispersion gradually became colorless (Figure S6), with essentially all $(>98 \%)$ of the trithiocarbonate chain-ends being destroyed within $8 \mathrm{~h}$ at $70{ }^{\circ} \mathrm{C}$ when using initiator/trithiocarbonate 


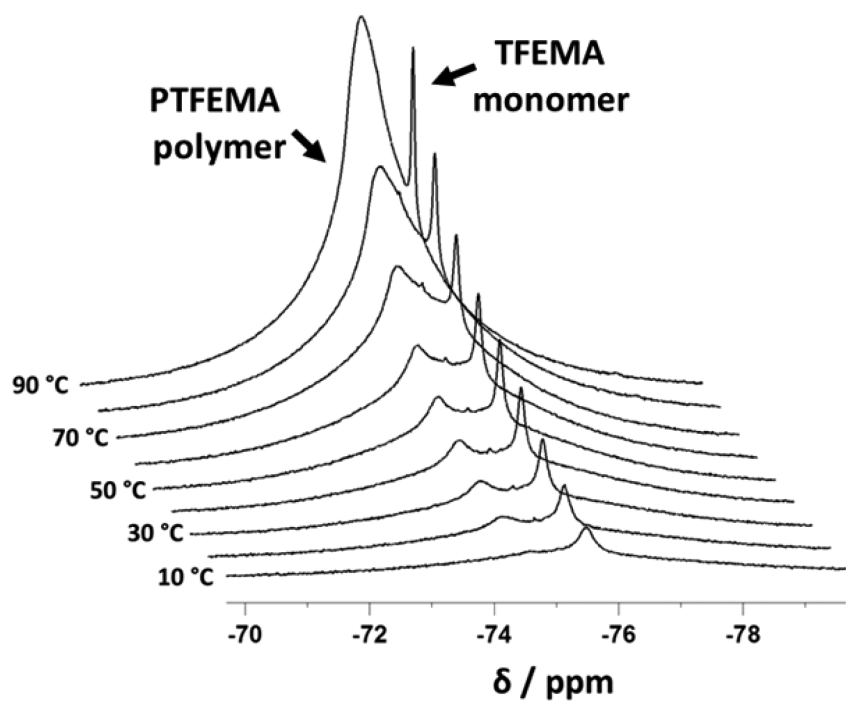

Figure 7. Variable-temperature ${ }^{19} \mathrm{~F}$ NMR spectra recorded from 10 to $90{ }^{\circ} \mathrm{C}$ for PSMA $_{12}-$ PTFEMA $_{98}$ nanoparticles obtained after $98 \%$ TFEMA conversion. The broad PTFEMA signal at $-74.6 \mathrm{ppm}$ observed at elevated temperature indicates ingress of hot solvent into the nanoparticle cores (note that the sharp signal observed at -75.3 ppm is assigned to the $2 \%$ residual TFEMA monomer).

molar ratios of either 7.5 or 10 (Figure 8). Moreover, successful removal of the aromatic ring associated with each trithiocarbonate end-group was confirmed by THF GPC analysis using a UV detector at a fixed wavelength of $260 \mathrm{~nm}$ in combination with a series of near-monodisperse polystyrene calibration standards (Figure 9 and Table S1). The PSMA $_{12}$ PTFEMA $_{98}$ diblock copolymer chains also absorb at this wavelength, but solely as a result of the aromatic character of their trithiocarbonate chain-ends. Hence, complete removal of these RAFT chain-ends leads to no GPC signal being observed (see red curve in Figure 9). Further experiments were performed to confirm that RAFT end-group removal had no significant effect on the nanoparticle morphology. TEM images confirmed that the original spherical morphology was retained (Figure 10a and $\mathrm{b}$ ), while SAXS patterns (fitted using a spherical micelle model ${ }^{69-71}$ ) recorded for a $1.0 \% \mathrm{w} / \mathrm{w}$ $\mathrm{PSMA}_{12}-\mathrm{PTFEMA}_{98}$ dispersion in $n$-tetradecane indicated minimal change in the volume-average diameter of the nanoparticle cores before $(25.5 \mathrm{~nm})$ and after $(24.9 \mathrm{~nm})$ RAFT chain-end removal (see Figure 10c and Table S2).

Finally, we note that the highly transparent dispersions described herein should offer new opportunities for further studies in the field of colloid science. ${ }^{54-56}$ This is because such isorefractive nanoparticles should exhibit significantly weaker attractive van der Waals interactions compared to nanoparticles for which the refractive index difference is not negligible, which in principle should lead to enhanced colloidal stability. ${ }^{72,73}$ Furthermore, isorefractive nanoparticles also enable tracer diffusion experiments to be performed at relatively high volume fractions. Moreover, there is the possibility of preparing new examples of highly transparent Pickering emuls ions, ${ }^{7-77}$ which will be explored in the near future.

\section{CONCLUSIONS}

RAFT dispersion polymerization of TFEMA enables the PISA synthesis of sterically stabilized PSMA-PTFEMA spherical
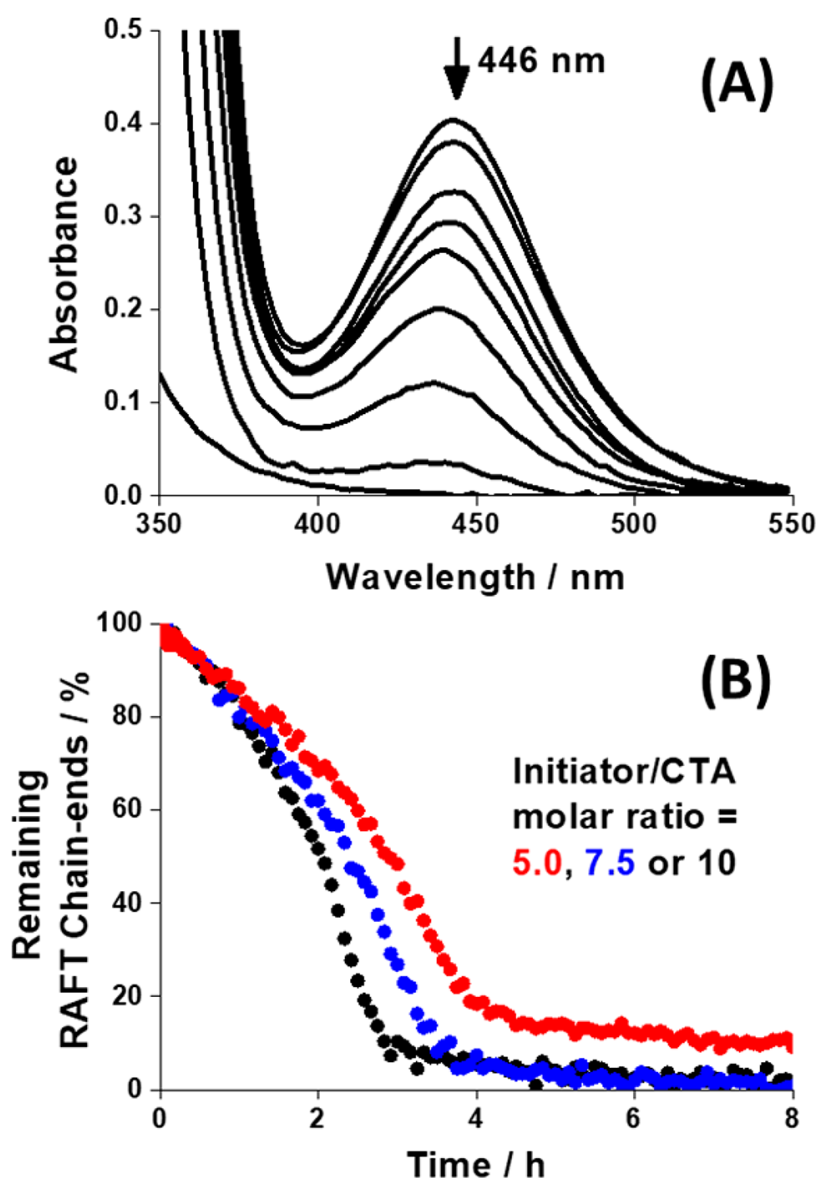

Figure 8. (a) In situ visible absorption spectra recorded at $70{ }^{\circ} \mathrm{C}$ for a $30 \% \mathrm{w} / \mathrm{w}$ dispersion of $\mathrm{PSMA}_{12}-\mathrm{PTFEMA}_{98}$ spherical nanoparticles in $n$-tetradecane using 7.5 equiv. lauroyl peroxide (Luperox) initiator to remove the trithiocarbonate end-groups. The $446 \mathrm{~nm}$ absorption band assigned to this RAFT end-group disappeared within $8 \mathrm{~h}$. (b) Kinetics of RAFT chain-end removal at $70{ }^{\circ} \mathrm{C}$ for this PISA formulation under the same conditions using lauroyl peroxide/RAFT chain-end molar ratios of 5.0 (red), 7.5 (blue), and 10 (black).

nanoparticles in $n$-alkanes at $70{ }^{\circ} \mathrm{C}$. In the case of $n$ tetradecane, the reaction solution is almost perfectly isorefractive at this temperature: this minimizes the problem of nanoparticle scattering and enables visible absorption spectra to be recorded in situ using a commercial optical immersion probe at up to $30 \% \mathrm{w} / \mathrm{w}$ solids. Unexpectedly, the polymerization kinetics can be conveniently monitored by examining the increase in absorbance of a relatively weak $\mathrm{n} \rightarrow$ $\pi^{*}$ band at $446 \mathrm{~nm}$. This is because the relatively large increase in density that occurs on converting TFEMA into PTFEMA leads to a significant volume contraction during the polymerization. This $446 \mathrm{~nm}$ band is assigned to the trithiocarbonate chain-ends and remains constant for at least $2 \mathrm{~h}$ under monomer-starved conditions, which indicates remarkably good pseudoliving character for such heterogeneous polymerizations. Gradual loss of the RAFT chain-ends is observed over longer time scales, as expected. This degradation can be accelerated by the addition of excess initiator, which leads to the complete loss of RAFT chain-ends within $8 \mathrm{~h}$ at $70{ }^{\circ} \mathrm{C}$, while preserving the original spherical morphology. This is consistent with ${ }^{19} \mathrm{~F}$ NMR studies, which indicate solventplasticized PTFEMA cores at this temperature. Such solvation is essential to allow ingress of radicals derived from the lauroyl 


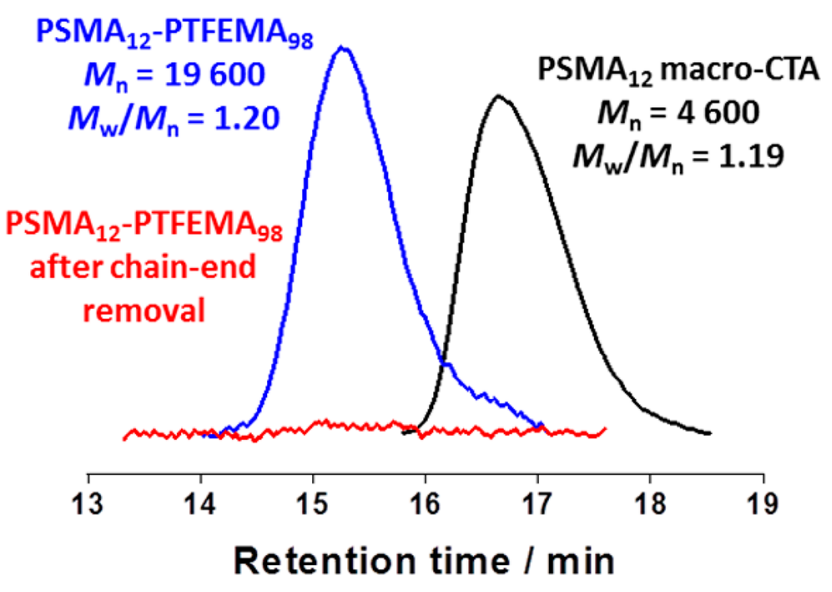

Figure 9. UV GPC traces recorded for a PSMA ${ }_{12}$ macro-CTA (black trace), as-prepared $\mathrm{PSMA}_{12}-\mathrm{PTFEMA}_{98}$ diblock copolymer (blue trace), and a $\mathrm{PSMA}_{12}-\mathrm{PTFEMA}_{98}$ diblock copolymer after removal of its RAFT end-groups (red trace). The UV detector wavelength was set at $260 \mathrm{~nm}$, and a series of polystyrene standards were used for calibration. The $\mathrm{PSMA}_{12}-\mathrm{PTFEMA}_{98}$ diblock copolymer chains also absorb at this wavelength, but solely as a result of the aromatic character of their trithiocarbonate chain-ends. Hence, complete removal of these RAFT chain-ends leads to no GPC signal being observed (see red trace).

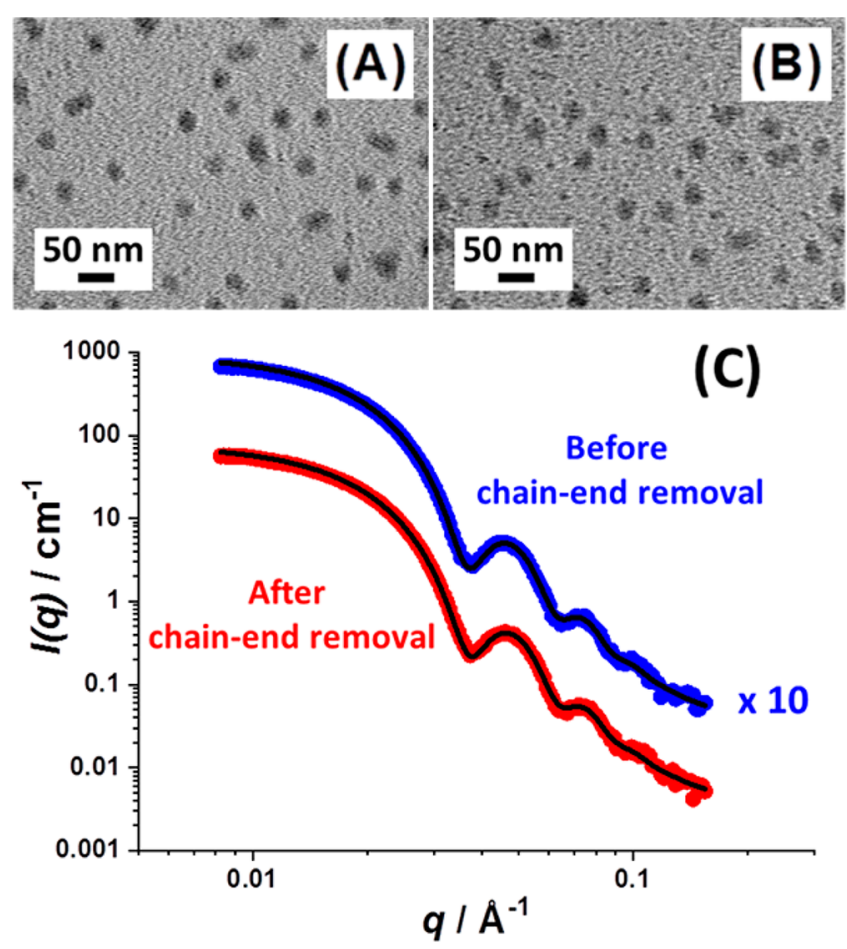

Figure 10. TEM images obtained for $\mathrm{PSMA}_{12}-\mathrm{PTFEMA}_{98}$ nanoparticles (a) before and (b) after RAFT chain-end removal at $70{ }^{\circ} \mathrm{C}$ using lauroyl peroxide (peroxide/trithiocarbonate molar ratio $=7.5$ ). (c) SAXS patterns recorded for $1.0 \% \mathrm{w} / \mathrm{w}$ dispersions of $\mathrm{PSMA}_{12}$ PTFEMA $_{98}$ nanoparticles in $n$-tetradecane before (blue) and after (red) RAFT chain-end removal under the same conditions. Fitting these patterns using a spherical micelle model yielded nanoparticle core diameters of $25.5 \mathrm{~nm}$ (before chain-end removal) and $24.9 \mathrm{~nm}$ (after chain-end removal).

peroxide initiator into the nanoparticle cores to remove the RAFT chain-ends. ${ }^{19} \mathrm{~F}$ NMR spectroscopy also enabled micellar nucleation to be monitored during the TFEMA polymerization, and remarkably good agreement is obtained for the kinetic data obtained with this latter technique compared to that determined using in situ visible absorption spectroscopy. This study has provided important new insights regarding the true nature of RAFT dispersion polymerization, which is a powerful and versatile technique for the rational design of a wide range of organic nanoparticles.

\section{ASSOCIATED CONTENT}

\section{S Supporting Information}

The Supporting Information is available free of charge on the ACS Publications website at DOI: 10.1021/jacs.8b07953.

Materials and instrumentation details, and synthesis protocols for preparation of the PSMA macro-CTA and PSMA-PTFEMA block copolymer dispersions; experimental details for the in situ visible absorption spectroscopy and ${ }^{19} \mathrm{~F}$ NMR studies; representative TEM images of PSMA $_{32}$-PTFEMA 490 spherical nanoparticles, digital photograph and schematic of the allquartz UV/visible immersion probe, equation to determine absorbance change due to volume contraction, equation to convert absorbance into monomer conversion, SAXS patterns recorded at intermediate TFEMA conversions when targeting PSMA $_{12}-$ PTFE$\mathrm{MA}_{100}$ nanoparticles, typical in situ ${ }^{19} \mathrm{~F}$ NMR spectrum, in situ ${ }^{19} \mathrm{~F}$ NMR kinetics, digital images of a transparent dispersion of PSMA $_{12}-$ PTFEMA $_{100}$ nanoparticles before and after chain-end removal, THF GPC data obtained using refractive index and UV detectors, and SAXS fitting parameters for a $\mathrm{PSMA}_{12}-\mathrm{PTFEMA}_{98}$ dispersion before and after RAFT chain-end removal (PDF)

\section{AUTHOR INFORMATION}

\section{Corresponding Author}

*s.p.armes@shef.ac.uk

ORCID ${ }^{\circ}$

Steven P. Armes: 0000-0002-8289-6351

Notes

The authors declare no competing financial interest.

\section{ACKNOWLEDGMENTS}

S.P.A. thanks EPSRC (Established Career Particle Technology Fellowship EP/R003009/1) and the ERC (Advanced Investigator Grant: PISA 320372) for financial support. Lubrizol is thanked for funding a Ph.D. studentship for E.J.C. and for permission to publish this research. Dr. S. Tzokov and Dr. C. Hill are thanked for their assistance with TEM analysis. Dr. C. Robertson and Dr. O. O. Mykhaylyk are thanked for their assistance with the ${ }^{19} \mathrm{~F}$ NMR studies and SAXS analyses, respectively.

\section{REFERENCES}

(1) Bates, C. M.; Bates, F. S. Macromolecules 2017, 50, 3.

(2) Mai, Y.; Eisenberg, A. Chem. Soc. Rev. 2012, 41, 5969.

(3) Moad, G.; Rizzardo, E.; Thang, S. H. Aust. J. Chem. 2012, 65, 985 .

(4) Moad, G.; Rizzardo, E.; Thang, S. H. Aust. J. Chem. 2009, 62, 1402.

(5) Moad, G.; Rizzardo, E.; Thang, S. H. Acc. Chem. Res. 2008, 41, 1133.

(6) Stenzel, M. H. Chem. Commun. 2008, 3486. 
(7) Zetterlund, P. B.; Gody, G.; Perrier, S. Macromol. Theory Simul. 2014, 23, 331.

(8) Gody, G.; Maschmeyer, T.; Zetterlund, P. B.; Perrier, S. Macromolecules 2014, 47, 3451.

(9) Carmean, R. N.; Becker, T. E.; Sims, M. B.; Sumerlin, B. S. Chem. 2017, 2, 93.

(10) Grazon, C.; Rieger, J.; Méallet-Renault, R.; Clavier, G.; Charleux, B. Macromol. Rapid Commun. 2011, 32, 699.

(11) Sponchioni, M.; Morosi, L.; Lupi, M.; Capasso Palmiero, U. RSC Adv. 2017, 7, 50981.

(12) Fu, C.; Huang, Z.; Hawker, C. J.; Moad, G.; Xu, J.; Boyer, C. Polym. Chem. 2017, 8, 4637.

(13) Cauet, S. I.; Wooley, K. L. J. Polym. Sci., Part A: Polym. Chem. 2010, 48, 2517.

(14) Lu, A.; Smart, T. P.; Epps, T. H.; Longbottom, D. A.; O’Reilly, R. K. Macromolecules 2011, 44, 7233.

(15) Postma, A.; Davis, T. P.; Li, G.; Moad, G.; O’Shea, M. S. Macromolecules 2006, 39, 5307.

(16) Touris, A.; Chanpuriya, S.; Hillmyer, M. A.; Bates, F. S. Polym. Chem. 2014, 5, 5551.

(17) Yin, L. G.; Lodge, T. P.; Hillmyer, M. A. Macromolecules 2012, 45, 9460.

(18) Ng, G.; Yeow, J.; Xu, J.; Boyer, C. Polym. Chem. 2017, 8, 2841.

(19) Chen, W. L.; Cordero, R.; Tran, H.; Ober, C. K. Macromolecules 2017, 50, 4089.

(20) Perrier, S. Macromolecules 2017, 50, 7433.

(21) Canning, S. L.; Smith, G. N.; Armes, S. P. Macromolecules 2016, 49, 1985.

(22) Derry, M. J.; Fielding, L. A.; Armes, S. P. Prog. Polym. Sci. 2016, $52,1$.

(23) Warren, N. J.; Armes, S. P. J. Am. Chem. Soc. 2014, 136, 10174.

(24) Charleux, B.; Delaittre, G.; Rieger, J.; D’Agosto, F. Macromolecules 2012, 45, 6753.

(25) Zetterlund, P. B.; Thickett, S. C.; Perrier, S.; Bourgeat-Lami, E.; Lansalot, M. Chem. Rev. 2015, 115, 9745.

(26) Blanazs, A.; Ryan, A. J.; Armes, S. P. Macromolecules 2012, 45, 5099.

(27) Jones, E. R.; Mykhaylyk, O. O.; Semsarilar, M.; Boerakker, M.; Wyman, P.; Armes, S. P. Macromolecules 2016, 49, 172.

(28) Jones, E. R.; Semsarilar, M.; Blanazs, A.; Armes, S. P. Macromolecules 2012, 45, 5091.

(29) Derry, M. J.; Fielding, L. A.; Armes, S. P. Polym. Chem. 2015, 6, 3054.

(30) Fielding, L. A.; Lane, J. A.; Derry, M. J.; Mykhaylyk, O. O.; Armes, S. P. J. Am. Chem. Soc. 2014, 136, 5790.

(31) Boisse, S.; Rieger, J.; Belal, K.; Di-Cicco, A.; Beaunier, P.; Li, M. H.; Charleux, B. Chem. Commun. 2010, 46, 1950.

(32) Tan, J.; Sun, H.; Yu, M.; Sumerlin, B. S.; Zhang, L. ACS Macro Lett. 2015, 4, 1249.

(33) Zhao, W.; Ta, H. T.; Zhang, C.; Whittaker, A. K. Biomacromolecules 2017, 18, 1145.

(34) Yeow, J.; Boyer, C. Advanced Science 2017, 4, 1700137.

(35) Ferguson, C. J.; Hughes, R. J.; Nguyen, D.; Pham, B. T. T.; Gilbert, R. G.; Serelis, A. K.; Such, C. H.; Hawkett, B. S. Macromolecules 2005, 38, 2191.

(36) Lowe, A. B. Polymer 2016, 106, 161.

(37) Zhang, W. J.; Hong, C. Y.; Pan, C. Y. Biomacromolecules 2017, $18,1210$.

(38) Roth, P. J.; Kessler, D.; Zentel, R.; Theato, P. J. Polym. Sci., Part A: Polym. Chem. 2009, 47, 3118.

(39) Roth, P. J.; Kessler, D.; Zentel, R.; Theato, P. Macromolecules 2008, 41, 8316.

(40) Willcock, H.; O'Reilly, R. K. Polym. Chem. 2010, 1, 149.

(41) Discekici, E. H.; Shankel, S. L.; Anastasaki, A.; Oschmann, B.; Lee, I.-H.; Niu, J.; McGrath, A. J.; Clark, P. G.; Laitar, D. S.; de Alaniz, J. R.; Hawker, C. J.; Lunn, D. J. Chem. Commun. 2017, 53, 1888.

(42) Semsarilar, M.; Jones, E. R.; Armes, S. P. Polym. Chem. 2014, 5, 195.
(43) Blackman, L. D.; Doncom, K. E. B.; Gibson, M. I.; O’Reilly, R. K. Polym. Chem. 2017, 8, 2860.

(44) Gody, G.; Maschmeyer, T.; Zetterlund, P. B.; Perrier, S. Macromolecules 2014, 47, 639.

(45) Herfurth, C.; Malo de Molina, P.; Wieland, C.; Rogers, S.; Gradzielski, M.; Laschewsky, A. Polym. Chem. 2012, 3, 1606.

(46) McCormick, C. L.; Lowe, A. B. Acc. Chem. Res. 2004, 37, 312.

(47) Matioszek, D.; Dufils, P.-E.; Vinas, J.; Destarac, M. Macromol. Rapid Commun. 2015, 36, 1354.

(48) Fairbanks, B. D.; Gunatillake, P. A.; Meagher, L. Adv. Drug Delivery Rev. 2015, 91, 141.

(49) Wang, Z.; He, J.; Tao, Y.; Yang, L.; Jiang, H.; Yang, Y. Macromolecules 2003, 36, 7446.

(50) Jesson, C. P.; Pearce, C. M.; Simon, H.; Werner, A.; Cunningham, V. J.; Lovett, J. R.; Smallridge, M. J.; Warren, N. J.; Armes, S. P. Macromolecules 2017, 50, 182.

(51) Chong, Y. K.; Moad, G.; Rizzardo, E.; Thang, S. H. Macromolecules 2007, 40, 4446.

(52) Gaynor, J.; Schueneman, G.; Schuman, P.; Harmon, J. P. J. Appl. Polym. Sci. 1993, 50, 1645.

(53) Thomas, D. B.; Convertine, A. J.; Hester, R. D.; Lowe, A. B.; McCormick, C. L. Macromolecules 2004, 37, 1735.

(54) Sun, J. Z.; Erickson, M. C. E.; Parr, J. W. J. Cosmet. Sci. 2005 , $56,253$.

(55) Hibberd, D. J.; Mackie, A. R.; Moates, G. K.; Penfold, R.; Watson, A. D.; Barker, G. C. Colloids Surf., A 2007, 301, 453.

(56) Husband, F. A.; Garrood, M. J.; Mackie, A. R.; Burnett, G. R.; Wilde, P. J. J. Agric. Food Chem. 2001, 49, 859.

(57) Mazurowski, M.; Gallei, M.; Rehahn, M. ACS Macro Lett. 2012, $1,1362$.

(58) Laib, J. P.; Mittleman, D. M. J. Infrared, Millimeter, Terahertz Waves 2010, 31, 1015.

(59) Note that isorefractivity is only required at some point after the onset of micellization when the growing diblock copolymer nanoparticles are sufficiently large to scatter light. Thus, in the early (prenucleation) stage where substantial unreacted TFEMA monomer (refractive index $=1.361$ at $20^{\circ} \mathrm{C}$ ) is present in the reaction solution, the mismatched refractive index under such conditions does not prevent in situ spectroscopic studies. However, isorefractivity is essential in the latter stages of the RAFT dispersion polymerization of TFEMA where there are $33 \mathrm{~nm}$ diameter nanoparticles present at up to $30 \% \mathrm{w} / \mathrm{w}$ solids.

(60) Buffle, J.; van Leeuwen, H. P. Environmental Particles; Lewis Publishers: New York, 1993.

(61) Shi, Y.; Liu, G.; Gao, H.; Lu, L.; Cai, Y. Macromolecules 2009, 42, 3917.

(62) Skrabania, K.; Miasnikova, A.; Bivigou-Koumba, A. M.; Zehm, D.; Laschewsky, A. Polym. Chem. 2011, 2, 2074.

(63) Yaochen, Z.; Qingquan, X.; Lemin, Z.; Zhirong, X.; Yan, S.; Wanzhong, R. J. Polym. Sci., Part B: Polym. Phys. 2014, 52, 1037.

(64) Akpinar, B.; Fielding, L. A.; Cunningham, V. J.; Ning, Y.; Mykhaylyk, O. O.; Fowler, P. W.; Armes, S. P. Macromolecules 2016, 49, 5160.

(65) Pepper, D. C. Trans. Faraday Soc. 1949, 45, 404.

(66) Kwak, Y.; Goto, A.; Tsujii, Y.; Murata, Y.; Komatsu, K.; Fukuda, T. Macromolecules 2002, 35, 3026.

(67) Blanazs, A.; Madsen, J.; Battaglia, G.; Ryan, A. J.; Armes, S. P. J. Am. Chem. Soc. 2011, 133, 16581.

(68) Pissuwan, D.; Boyer, C.; Gunasekaran, K.; Davis, T. P.; Bulmus, V. Biomacromolecules 2010, 11, 412.

(69) Pedersen, J. S. J. Appl. Crystallogr. 2000, 33, 637.

(70) Pedersen, J. S.; Gerstenberg, M. C. Macromolecules 1996, 29, 1363.

(71) Pedersen, J. S.; Schurtenberger, P. Macromolecules 1996, 29, 7602.

(72) Bergstrom, L. Adv. Colloid Interface Sci. 1997, 70, 125.

(73) Israelachvili, J. N. Intermolecular and Surface Forces, 3rd ed.; Elsevier: New York, 2011. 
(74) Goodwin, J. W.; Ottewill, R. H.; Parentich, A. J. Phys. Chem. 1980, 84, 1580.

(75) Kopswerkhoven, M. M.; Fijnaut, H. M. J. Chem. Phys. 1982, 77, 2242

(76) Kopswerkhoven, M. M.; Pathmamanoharan, C.; Vrij, A.; Fijnaut, H. M. J. Chem. Phys. 1982, 77, 5913.

(77) Rymaruk, M. J.; Thompson, K. L.; Derry, M. J.; Warren, N. J.; Ratcliffe, L. P. D.; Williams, C. N.; Brown, S. L.; Armes, S. P. Nanoscale 2016, 8, 14497. 